\title{
Diretrizes
}

\section{Sopros cardíacos, valvopatias, próteses valvares e endocardite}

\section{1 - Sopros cardíacos}

As características dos sopros cardíacos, particularmente em doenças valvares ou congênitas, geralmente permitem ao clínico concluir sobre a possível doença envolvida responsável pelo sopro, embora algumas vezes lesões valvares significantes podem não produzir sopros audíveis ${ }^{1,2,3}$. Sopros conhecidos como inocentes têm características próprias e usualmente não necessitam da ecocardiografia. Em sopros com características duvidosas ou em pacientes em que a ausculta cardíaca é difícil, a Doppler ecocardiografia é a técnica mais recomendada e pode ser usada para o diagnóstico definitivo do tipo de sopro $^{4}$. Devido à alta sensibilidade do método, insuficiência mínima em valva com aspecto morfológico e funcional normal pode ser encontrada em indivíduos normais, principalmente nas valvas tricúspide, pulmonar, mitral e mesmo na aórtica. Usualmente não representam doença cardíaca nem justificam sopros cardíacos, mesmo os inocentes.

\section{Tabela 8 - Recomendações do ecocardiograma em pacientes com sopro}

\begin{tabular}{lc}
\hline Recomendações & Classe \\
\hline $\begin{array}{l}\text { Pacientes assintomáticos com sopros indicativos de alta } \\
\text { probabilidade de doença cardiaca }\end{array}$ & I \\
\hline $\begin{array}{l}\text { Pacientes com sopro, sem sintomas e com baixa probabilidade de } \\
\text { doença cardíaca, mas que não podem podem ser excluídos apenas } \\
\text { pela clínica, eletrocardiograma, radiografia de tórax. }\end{array}$ & Ila \\
\hline $\begin{array}{l}\text { Pacientes adultos sem sintomas, com sopro cujas características } \\
\text { sugerem ser funcional ou inocente }\end{array}$ & III \\
\hline
\end{tabular}

Tabela 9 - Recomendações para ecocardiografia na EM

\begin{tabular}{lc}
\hline Recomendações & Classe \\
\hline Diagnóstico, avaliação da gravidade e da repercussão & I \\
\hline $\begin{array}{l}\text { Avaliação da morfologia valvar para determinar a possibilidade de } \\
\text { tratamento por meio da valvoplastia percutânea }\end{array}$ & I I \\
\hline Diagnóstico e avaliação de lesão valvar associada & I \\
\hline $\begin{array}{l}\text { Reavaliação de pacientes com EM e mudanças dos sinais ou } \\
\text { sintomas }\end{array}$ & I \\
\hline $\begin{array}{l}\text { Avaliação das alterações hemodinâmicas e adaptação ventricular } \\
\text { durante gravidez }\end{array}$ & Ila \\
\hline $\begin{array}{l}\text { Ecocardiografia sob estresse para avaliar pressão pulmonar e } \\
\text { gradientes de pressão transvalvar em pacientes com discrepância } \\
\text { entre sintomas e gravidade da estenose em repouso }\end{array}$ \\
\hline $\begin{array}{l}\text { Reavaliação anual da pressão da AP em pacientes assintomáticos } \\
\text { com lesão importante }\end{array}$
\end{tabular}

\section{2 - Valvas nativas}

A Doppler ecocardiografia é uma técnica diagnóstica fundamental na avaliação e prognóstico dos pacientes com doença valvar ${ }^{4}$, permitindo analisar a etiologia, os mecanismos envolvidos e a anatomia funcional das lesões de insuficiência e de estenose que facilitam a decisão terapêutica e o eventual procedimento cirúrgico, se necessário. Com as diferentes técnicas de Doppler espectral, pulsátil e contínuo, e de mapeamento de fluxo em cores a sensibilidade para detecção de estenose ou refluxo valvar é alta ${ }^{5}$ e também podem ser obtidos parâmetros quantitativos, como área valvar, gradientes de pressão, máximo e médio, e outros índices, que estabelecem a gravidade ${ }^{6}$. O exame permite ainda analisar a repercussão funcional da lesão sobre as câmaras cardíacas e a função ventricular, bem como estimar a pressão arterial pulmonar.

\subsection{1 - Valvopatias mitrais}

\subsubsection{1 - Estenose mitral}

O eco transtorácico associado ao Doppler espectral define as alterações estruturais da estenose mitral (EM), caracterizando o envolvimento valvar e subvalvar, estabelecendo os elementos necessários para indicação de valvotomia por cateter balão; permite o cálculo da área efetiva de fluxo, dos gradientes diastólicos médio e máximo e identifica lesões associadas (refluxos valvares, trombos atriais, hipertensão pulmonar). $\mathrm{O}$ exame transesofágico possibilita a identificação de trombos não diagnosticados ao exame de superfície, sobretudo com localização no apêndice atrial.

O eco com esforço físico ou sob estresse com dobutamina tem sido também empregado em pacientes com doença valvar para avaliação hemodinâmica, principalmente quando há discordância entre a clínica e a Doppler ecocardiografia em repouso. Em pacientes com estenose mitral (EM), a Doppler ecocardiografia sob esforço físico permite analisar o comportamento dos gradientes de pressão transvalvar e a pressão sistólica pulmonar. Elevação da pressão sistólica pulmonar acima de $60 \mathrm{mmHg}$ pode ser considerado um critério de indicação cirúrgica ${ }^{3}$.

\subsubsection{2 - Insuficiência mitral}

Na insuficiência mitral (IM), os parâmetros que definem a gravidade são as medidas da área do jato de refluxo, do diâmetro da vena contracta e cálculos da área do orifício e do volume de refluxo, obtidos pela ecocardiografia Doppler, incluindo mapeamento de fluxo em $\operatorname{cores}^{5,7}$ e, eventualmente, ecocardiografia tridimensional ${ }^{8}$. Além da importância desses parâmetros, medidas dos diâmetros ou volumes ventriculares e cavidades atriais da FE do VE e da pressão pulmonar obtidas pelas mesmas técnicas ecocardiográficas são importantes no prognóstico e decisão terapêutica dos pacientes com ou sem sintomas ${ }^{3}$. A ecocardiografia tridimensional facilita a visibilização espacial das valvas e suas anormalidades estruturais e proporciona maior precisão na análise da função do VE, importante nos pacientes com IM $\mathrm{M}^{9-12}$. Alguns estudos já demonstraram ótimos resultados nas medidas da área valvar mitral e estimativa do refluxo mitral ${ }^{8}$. 
Se o diagnóstico ou a quantificação das lesões valvares não podem ser estabelecidos de maneira satisfatória devido à limitação da qualidade das imagens pelo exame transtorácico

\section{Tabela 10 - Recomendaçoes para ETE na EM}

\begin{tabular}{lc}
\hline Recomendações & Classe \\
\hline $\begin{array}{l}\text { Em candidatos para valvoplastia percutânea para pesquisa de } \\
\text { trombo e avaliação do grau de insuficiência mitral }\end{array}$ & । \\
\hline Em pacientes com imagem transtorácica inadequada & । \\
\hline $\begin{array}{l}\text { Orientação durante procedimento intervencionista (valvoplastia } \\
\text { percutânea) }\end{array}$ & ॥la \\
\hline $\begin{array}{l}\text { Para avaliar morfologia valvar e hemodinâmica com } \\
\text { ecocardiografia transtorácia satisfatória }\end{array}$ & III \\
\hline
\end{tabular}

\section{Tabela 11 - Recomendações para ecocardiografia transtorácia} na IM

\begin{tabular}{lc}
\hline Recomendações & Classe \\
\hline Avaliação inicial da gravidade e mecanismo da IM & । \\
\hline $\begin{array}{l}\text { Avaliação anual das dimensões e da função do VE em pacientes } \\
\text { com IM moderada a grave sem mudanças de sintomas }\end{array}$ & । \\
\hline Pacientes com IM e modificação dos sinais ou sintomas & । \\
\hline Avaliação no primeiro mês pós-operatório & । \\
\hline $\begin{array}{l}\text { Avaliação das alterações hemodinâmicas e adaptação ventricular } \\
\text { durante gestação }\end{array}$ & Ila \\
\hline $\begin{array}{l}\text { Ecocardiografia sob esforço em pacientes assintomáticos com } \\
\text { IM grave para avaliar tolerância ao esforço e efeitos na pressão } \\
\text { pulmonar }\end{array}$ & III \\
\hline $\begin{array}{l}\text { Avaliação rotineira de IM discreta com função e dimensões normais } \\
\text { do VE }\end{array}$ & \\
\hline
\end{tabular}

\section{Referências}

1. Miyake T, Yokoyama T. Evaluation of transient heart murmur resembling pulmonary artery stenosis in term infants by Doppler and M-mode echocardiography. Jpn Circ J. 1993; 57: 77-83.

2. Auerback ML. High tech in a low tech country. West J Med. 1993; 159: 93-4.

3. Bonow RO, Carabello BA, Chatterjee K, de Leon Jr AC, Halperin JL, Hiratzka LF, et al. ACC/AHA 2006 guidelines for the management of patients with valvular heart disease. J Am Coll Cardiol. 2006; 48: 1-148.

4. Cheitlin MD, Antman EM, Smith SC Jr, Armstrong WF, Aurigemma GP, Beller GA, etal. ACC/AHA/ASE 2003 guideline update for the clinical application of echocardiography. J Am Soc Echocardiogr. 2003 Oct; 16 (10): 1091-110.
(ETT), a complementação com ecocardiografia transesofágica (ETE) é fundamental ${ }^{13}$.

\section{Tabela 12 - Recomendações para ETE em pacientes com IM}

\begin{tabular}{lc}
\hline Recomendações & Classe \\
\hline $\begin{array}{l}\text { Avaliação intraoperatória para definir o mecanismo da IM e auxiliar } \\
\text { no reparo valvar }\end{array}$ & I \\
\hline $\begin{array}{l}\text { ETT insatisfatório para a determinação da gravidade e/ou do } \\
\text { mecanismo da insuficiência, ou para a avaliação da função do VE }\end{array}$ & । \\
\hline $\begin{array}{l}\text { Pacientes assintomáticos com IM grave para avaliar a } \\
\text { possibilidade de reparo valvar }\end{array}$ & Ila \\
\hline Avaliação periódica de pacientes com IM discreta
\end{tabular}

Tabela 13 - Recomendações para ecocardiografia em pacientes com prolapso da valva mitral (PVM)

\begin{tabular}{lc}
\hline Recomendações & Classe \\
\hline $\begin{array}{l}\text { Diagnóstico, avaliação anatômica e funcional em pacientes com } \\
\text { sinais físicos de PVM }\end{array}$ & । \\
\hline $\begin{array}{l}\text { Confirmação do PVM em pacientes com diagnóstico prévio, mas } \\
\text { sem evidências clínicas que o suportem }\end{array}$ & Ila \\
\hline $\begin{array}{l}\text { Estratificação de risco em pacientes com clínica ou diagnóstico } \\
\text { de PVM }\end{array}$ & IIb \\
\hline $\begin{array}{l}\text { Exclusão de PVM em parentes de 10 grau em pacientes com } \\
\text { doença valvar mixomatosa }\end{array}$ & III \\
\hline $\begin{array}{l}\text { Exclusão de PVM em pacientes sem sinais físicos sugestivos ou } \\
\text { história familiar }\end{array}$ & \\
\hline $\begin{array}{l}\text { Ecocardiogramas periódicos em pacientes com PVM sem } \\
\text { insuficiência ou com insuficiência de grau discreto, sem alterações } \\
\text { de sintomas ou sinais clínicos }\end{array}$ &
\end{tabular}

5. Zoghbi WA, Enriquez-Sarano M, Foster E, Grayburn PA, Kraft CD, Levine RA, et al. Recommendations for evaluation of the severity of native valvular regurgitation with two-dimensional and Doppler echocardiography. J Am Soc Echocardiogr. 2003; 16: 777-802.

6. Otto CM, Pellikka PA, Quiñones M, Baumgartner H, Hung J, Bermejo J, et al Echocardiographic assessment of valve stenosis: EAE/ASE recommendations for clinical practice. J Am Soc Echocardiograph. 2009; 22 (1): 1-23.

7. Pinheiro AC, Mancuso FJ, Hemerly DF, Kiyose AT, Campos O, de Andrade JL, et al. Diagnostic value of color flow mapping and Doppler echocardiography in the quantification of mitral regurgitation in patients with mitral valve prolapse or rheumatic heart disease. J Am Soc Echocardiogr. 2007 Oct; 20 (10): 1141-8. 


\section{Diretrizes}

8. Little SH, Pirat B, Kumar R, Igo SR, McCulloch M, Hartley CJ, et al. Threedimensional color Doppler echocardiography for direct measurement of vena contracta area in mitral regurgitation: in vitro validation and clinical experience. J Am Coll Cardiol Img. 2008; 1(6): 695-704.

9. Sharma R, Mann I, Drummond L, Livesey SA, Simpson IA. The evaluation of real-time 3-dimensional transthoracic echocardiography for the preoperative functional assessment of patients with mitral valve prolapse: a comparison with 2-dimensional transesophageal echocardiography. J Am Soc Echocardiogr. 2007; 20 (8): 934-40.

10. Sugeng L, Coon P, Weinert L, Jolly N, Lammertin G, BednarzJE, et al. Use of real-time 3-dimensional transthoracic echocardiography in the evaluation of mitral valve disease. J Am Soc Echocardiogr. 2006; 19 (4): 413-21.
11. Chu JW, Levine RA, Cha S, Poh KK, Morris E, Hua L, et al. Assessing mitral valve area and orifice geometry in calcific mitral stenosis: a new solution by real-time three-dimensional echocardiography. J Am Soc Echocardiogr. 2008; 21 (9): 1006-9.

12. Zamorano I, Perez de Isla L, Sugeng L, Cordeiro P, Rodrigo IL, Almeria C, et al. Noninvasive assessment of mitral valve area during percutaneos balloon mitral valvuloplasty: role of real-time 3D echocardiography. Eur Heart J. 2004; 25 (23): 2086-91.

13. Enriquez-Sarano M, Freeman WK, Tribouilly CM, Orszulak TA, Khanderia BJ, Seward JB, et al. Functional anatomy of mitral regurgitation: accuracy and outcome implications of transesophageal echocardiography. J Am Coll Cardiol. 1999; 34 (4): 1129-36.

\subsection{2 - Valvopatias aórticas}

\subsubsection{1 - Estenose aórtica}

A ecocardiografia é o método padrão para avaliação da gravidade da estenose valvar aórtica (EAo). O estudo hemodinâmico está recomendado para os casos de discrepância com a clínica ou quando o ecocardiograma não for diagnóstico ${ }^{1-3}$.

A avaliação anatômica da valva aórtica permite, muitas vezes, a definição da causa da doença valvar (congênita, reumática ou degenerativa). Baseia-se na associação de

\section{Tabela 14 - Recomendações do ecocardiograma nos pacientes com EAo}

\begin{tabular}{lc}
\hline Recomendações & Classe \\
\hline Diagnóstico e avaliação da gravidade da EAo & । \\
\hline $\begin{array}{l}\text { Pacientes com estenose valvar aórtica para a avaliação da } \\
\text { espessura de parede, tamanho e função do VE }\end{array}$ & । \\
\hline $\begin{array}{l}\text { Reavaliação de pacientes com o diagnóstico de EAo com mudança } \\
\text { de sintomas ou sinais }\end{array}$ & । \\
\hline $\begin{array}{l}\text { Avaliação de mudanças na gravidade hemodinâmica e função do } \\
\text { VE nas pacientes com diagnóstico de EAo durante a gravidez }\end{array}$ & । \\
\hline Reavaliação anual dos pacientes assintomáticos com EAo & । \\
\hline
\end{tabular}

Tabela 15 - Recomendações do ecocardiograma sob estresse na EAo

\begin{tabular}{lc}
\hline Recomendações & Classe \\
\hline $\begin{array}{l}\text { Ecocardiografia sob estresse com dobutamina para avaliar EAo } \\
\text { com baixo fluxo e baixo gradiente com disfunção do VE }\end{array}$ & ॥la \\
\hline $\begin{array}{l}\text { Ecocardiograma de esforço em assintomáticos com EAo para } \\
\text { avaliar sintomas induzidos pelo exercício e respostas anormais de } \\
\text { pressão ou comportamento dos gradientes }\end{array}$ & ॥lb \\
\hline Ecocardiograma de esforço em EAo sintomática & III \\
\hline
\end{tabular}

imagens dos vários cortes para identificação do número, mobilidades, espessura e presença de calcificação dos folhetos. As imagens e o Doppler permitem determinar o nível de obstrução (valvar, subvalvar ou supravalvar). As imagens transtorácicas geralmente são adequadas, embora a ETE possa ser útil quando as imagens são subótimas. O grau de calcificação é preditor de evolução clínica ${ }^{4,5}$. A estenose valvar aórtica reumática é caracterizada pela fusão comissural que resulta no orifício triangular com espessamento e calcificação mais proeminentes nas bordas das cúspides. Em tal situação, a ETE costuma ser superior ao ETT.

Tabela 16 - Recomendações do ecocardiograma nos pacientes com valva aórtica bicúspide e dilatação da aorta ascendente

\begin{tabular}{lc}
\hline Recomendações & Classe \\
\hline $\begin{array}{l}\text { Pacientes com valva aórtica bicúspide para a avaliação do } \\
\text { diâmetro da raiz da aorta e da aorta ascendente }\end{array}$ & । \\
\hline $\begin{array}{l}\text { Avaliação anual na valva aórtica bicúspide com dilatação da raiz } \\
\text { da aorta ou aorta ascendente (diâmetro maior que } 4,0 \mathrm{~cm})\end{array}$ & \\
\hline
\end{tabular}

\section{Tabela 17 - Recomendações para o ecocardiograma na IAo}

\begin{tabular}{lc}
\hline Recomendações & Classe \\
\hline $\begin{array}{l}\text { Confirmar a presença, etiologia e gravidade da IAo aguda ou } \\
\text { crônica }\end{array}$ & I \\
\hline $\begin{array}{l}\text { Dilatação da raiz da aorta para avaliar o grau de insuficiência e a } \\
\text { severidade da dilatação da aorta }\end{array}$ & I \\
\hline $\begin{array}{l}\text { Reavaliação periódica anual do tamanho e função do VE na IAo } \\
\text { grave e assintomática }\end{array}$ & I \\
\hline $\begin{array}{l}\text { Reavaliar IAo quando há novos sintomas ou evidente piora dos } \\
\text { existentes }\end{array}$ & \\
\hline
\end{tabular}


A gravidade da EAo pelas diretrizes europeias (ESC) e americanas (ACC/AHA) é: leve, moderada e grave. Em geral, os parâmetros ao Doppler ecocardiograma (velocidade máxima, gradiente médio e área valvar), em conjunto com os dados clínicos, avaliação da insuficiência valvar aórtica e função do VE, são suficientes para a decisão clínica. Nos casos de disfunção ventricular severa, medidas adicionais podem ser úteis, como se observa na Tabela 15. Os valores limites devem ser vistos com atenção, pois nenhum valor isolado é suficiente para definir conduta ${ }^{1,2}$.

\subsubsection{2 - Insuficiência aórtica}

O Doppler ecocardiograma é o método de escolha para detecção não invasiva, avaliação da severidade e da etiologia da insuficiência aórtica (IAo). São várias as etiologias da IAo, como degeneração valvar, calcificação, fibrose ou infecção, alteração do suporte do aparelho valvar ou dilatação do anel valvar $^{1,6}$. A avaliação qualitativa e medidas semiquantitativas são usadas regularmente, mas medidas quantitativas consomem tempo e são usadas mais seletivamente.

\section{Referências}

1. Bonow RO, Carabello BA, Chatterjee K, de Leon CC Jr, Faxon DP, Freed MD, et al. ACC/AHA 2006 Guidelines for the management of patients with valvular heart disease: a report of the American College of Cardiology/American Heart Association Task Force on Practice Guidelines (writing Committee to Revise the 1998 guidelines for the management of patients with valvular heart disease) developed in collaboration with the Society of Cardiovascular Anesthesiologists endorsed by the Society for Cardiovascular Angiography and Interventions and the Society of Thoracic Surgeons. J Am Coll Cardiol. 2006; 48: e1-148.

2. Vahanian A, Baumgartner H, Bax J, Butchart E, Dion R, Filippatos G, et al. Guidelines on the management of valvular heart disease: the task force on the management of valvular heart disease of the European Society of Cardiology. Eur Heart J. 2007; 28: 230-68.

\subsection{3 - Valvopatias tricúspide e pulmonar}

A ecocardiografia Doppler deve ser indicada nesses tipos de disfunção valvar para confirmar o diagnóstico, auxiliar na identificação da etiologia e dos mecanismos das lesões, determinar a gravidade, avaliar a pressão pulmonar, bem como as dimensões das cavidades cardíacas e a função do ventrículo direito. Assim como para as disfunções das demais valvas, tais indicações poderiam ser consideradas classe I, embora nenhuma referência clara exista em outras diretrizes nacionais ou internacionais ${ }^{1,2}$. Da mesma forma, as repetições de rotina da ecocardiografia Doppler no seguimento desses pacientes deveriam seguir as orientações descritas para as disfunções das valvas cardíacas esquerdas. A existência de insuficiência tricúspide (IT) importante associada à lesão valvar significativa identificada à ecocardiografia, como retração ou destruição de parte das cúspides, representa importante marcador de necessidade de substituição da valva. Contudo, na maioria dos pacientes com IT importante, esta é secundária à dilatação ventricular direita pela hipertensão pulmonar, estenose valvar pulmonar, cardiomiopatias, entre outras. A indicação ou não de correção com anuloplastia é ainda controversa. Atualmente, o momento da indicação é simultâneo à correção da doença da valva mitral. A ecocardiografia Doppler ajuda no diagnóstico e avaliação e, portanto, pode auxiliar no momento de correção. A associação de medidas da pressão sistólica pulmonar com medidas da circunferência do anel valvar podem ajudar na avaliação clínica e indicação de troca
3. Chambers J, Bach D, Dumesnil J, Otto C, Shah P, Thomas J. Crossing the aortic valve in severe aortic stenosis: no longer acceptable? J Heart Valve Dis. $2004 ; 13$ (3): 344-6.

4. Roberts WC, Ko JM. Frequency by decades of unicuspid, bicuspid, and tricuspid aortic valves in adults having isolated aortic valve replacement for aortic stenosis, with or without associated aortic regurgitation. Circulation. 2005; 111: 920-5.

5. Nistri S, Sorbo MD, Marin M, Palisi M, Scognamiglio R, Thiene G. Aortic root dilatation in young men with normally functioning bicuspid aortic valves. Heart. 1999; 82: 19-22

6. Rosenhek R, Binder T, Porenta G, Lang I, Christ G, Schemper M, et al Predictors of outcome in severe, asymptomatic aortic stenosis. N Engl J Med. 2000; 343: 611-7.

valvar. Diâmetro do anel tricúspide superior a duas vezes o normal $(>70 \mathrm{~mm}$ ) pode ser um determinante de necessidade de cirurgia. Essa avaliação parece ser melhor com o uso da ecocardiografia tridimensional e dados do exame transtorácico podem ser mais detalhados pela ecocardiografia transesofágica intraoperatória.

\subsection{4 - Lesões valvares múltiplas}

Uma variedade grande de combinações de lesões valvares pode ocorrer particularmente em pacientes com doença valvar de causa reumática. São exemplos as duplas disfunções das valvas mitral ou aórtica ou associações, como estenose mitral com insuficiência das valvas aórtica ou tricúspide. Cada disfunção pode ter influência hemodinâmica sobre a outra. Num paciente com dupla disfunção da valva mitral com insuficiência significativa, por exemplo, o gradiente de pressão transvalvar é usualmente maior do que seria na ausência da insuficiência e causa mais sintomas. As indicações da ecocardiografia nesses pacientes podem, portanto, ser mais frequentes do que nos pacientes com disfunções únicas e isoladas, mas devem, em geral, seguir as mesmas diretrizes. A análise quantitativa é a mais requisitada para determinar a importância relativa de cada disfunção. Da mesma forma que nas lesões isoladas, a ecocardiografia transesofágica pode ser necessária para um diagnóstico mais preciso na situação ambulatorial, bem como na avaliação intraoperatória'. 


\section{Diretrizes}

\section{Referências}

1. Bonow RO, Carabello BA, Chatterjee K, de Leon Jr AC, Halperin JL, Hiratzka LF, et al. ACC/AHA 2006 guidelines for the management of patients with valvular heart disease. J Am Coll Cardiol. 2006; 48: 1-148.

2. Cheitlin MD, Antman EM, Smith SC Jr, Armstrong WF, Aurigemma GP, Beller GA, et al. ACC/AHA/ASE 2003 guideline update for the clinical application of echocardiography. J Am Soc Echocardiogr. 2003 Oct; 16 (10): 1091-110.
3. Rogers JH, Bolling SF. The tricuspid valve: current perspective and evolving management of tricuspid regurgitation. Circulation. 2009; 119: 2718-25.

4. Dreyfus GD, Corbi PJ, Chan KM, Bahrami T. Secondary tricuspid regurgitation or dilatation: which should be the criteria for surgical repair? Ann Thorac Surg. 2005; 79: 127-32.

5. Anwar AM, Geleijnse ML, Cate FJ, Meijboom FJ. Assessment of tricuspid valve annulus size, shape and function using real-time three-dimensional echocardiography. Interact Cardiovasc Thorac Surg. 2006; 5: 683-7.

\section{3 - Próteses valvares}

A Doppler ecocardiografia, com suas modalidades transtorácica (ETT) e transesofágica (ETE), é um importante método diagnóstico não invasivo das disfunções protéticas.

É recomendado um ETT basal, a ser realizado entre 2-4 semanas após alta hospitalar da substituição valvar, tempo necessário para o coração se adaptar às novas condições hemodinâmicas. Nesse exame, é importante registrar as dimensões cavitárias, função ventricular, gradientes protéticos, áreas valvares, presença de refluxos funcionais ou patológicos etc., pois o procedimento será tomado como referência para exames evolutivos.

Em relação à periodicidade dos ecos evolutivos em portadores de próteses, não existe um consenso, sendo que a maioria acha não ser necessário realização de novo ecocardiograma em pacientes com próteses mecânicas, a não ser que ocorra alguma suspeita clínica de disfunção da prótese ou mudança na ausculta cardíaca. Um grupo menor sugere consultas e ETT anuais em pacientes portadores de qualquer tipo de próteses, mesmo sem sinais de disfunção, e a qualquer momento quando ocorrer suspeita clínica de disfunção da prótese, podendo haver complementação com a ETE, principalmente se houver suspeita de endocardite infecciosa ou trombose ${ }^{1}$. Nos casos de refluxo das próteses, é recomendado realizar ETT evolutivos a cada 3-6 meses $^{2}$.

O ecocardiograma é o método adequado para o diagnóstico das disfunções das próteses, tais como refluxos, estenoses, defeitos estruturais dos folhetos ou disco(s), presença de trombos ou fibrina ou endocardite infecciosa (Tabela 18) ${ }^{1}$. As próteses valvares, principalmente as mecânicas, causam reverberações e sombras acústicas, e sua análise está frequentemente prejudicada, principalmente pelo ETT. A ecocardiografia transesofágica, devido a sua proximidade e abordagem posterior do coração, consegue melhor acurácia diagnóstica nas disfunções das próteses valvares. Na realidade, ETT e ETE se complementam, sendo aconselhável sempre realizar o ETT completo e cuidadoso antes de se indicar a ETE.

O diagnóstico das estenoses das próteses deve ser sempre realizado com a utilização do Doppler ecocardiograma. Os fluxos das próteses são variáveis de acordo com cada modelo, podendo existir jatos excêntricos que podem causar falsas velocidades ao Doppler ecocardiograma. Os gradientes transprotéticos serão variáveis em cada modelo e tamanho, podendo existir gradientes elevados nos casos de próteses pequenas, mesmo que normofuncionantes, quando implantadas em pacientes de grande superfície corporal achado conhecido como paciente/prótese mismatch ${ }^{3}$. Por

Tabela 18 - Recomendações do ETT ou ETE nas próteses valvares

\begin{tabular}{l}
\hline Recomendações \\
\hline $\begin{array}{l}\text { ETT em pacientes com próteses valvares com alteração dos sinais ou sintomas clínicos que sugiram disfunção protética (estenose ou } \\
\text { regurgitação); ETE complementar pode estar indicada }\end{array}$ \\
\hline ETE em pacientes com ETT de disfunção protética, para confirmar diagnóstico e quantificar melhor a disfunção \\
\hline $\begin{array}{l}\text { Reavaliação periódica em pacientes portadores de próteses, com leve ou moderada disfunção ventricular sem modificação dos sintomas ou } \\
\text { sinais clínicos }\end{array}$ \\
\hline Reavaliação periódica em próteses valvares sem sinais ou sintomas de disfunção valvar \\
\hline Ila
\end{tabular}


outro lado, pacientes que permanecem com hipertrofia importante do VE no pós-operatório também podem apresentar gradientes elevados após o implante de prótese aórtica. Desse modo, é sempre importante a comparação com o ecocardiograma basal. Nas próteses biológicas, a causa mais frequente de estenose é a degeneração e calcificação dos folhetos, em geral uma complicação tardia. Nas próteses mecânicas, o crescimento de tecido fibroso para dentro do anel é conhecido como pannus, sendo também uma complicação tardia que pode causar estenose, refluxo ou dupla-disfunção protética.

A detecção e quantificação das regurgitações das próteses geralmente são dificultadas pela sombra acústica causada pelas próteses mecânicas, principalmente em posição mitral. Nesses casos, a ETE pode ajudar a detectar, quantificar e determinar se a incompetência é protética ou periprotética, funcional ou patológica. Devemos ter cuidado em diferenciar os refluxos "fisiológicos", que são comuns nas próteses, dos patológicos ${ }^{4}$.

Nos casos de suspeita de endocardite infecciosa em próteses, o diagnóstico é dificultado pela presença de sombras e reverberações, permitindo que o ETT consiga identificar geralmente apenas as grandes vegetações. Nessa suspeita clínica, é sempre recomendável realizar a ETE, que tem maior sensibilidade, podendo detectar vegetações menores e possíveis complicações, como abscessos anulares (Tabela 19)

Nos casos de fenômenos embólicos ou quadro de estenose aguda da prótese, principalmente em posição mitral, a suspeita de trombose valvar ou presença de strands (fibrina) deve ser suspeitada ${ }^{6}$, estando o ETT e a ETE indicados, com a finalidade de evitar a sombra acústica e melhor visualizar o AE e a face atrial da prótese. Nesses casos, além de procurar trombos ou fibrina na válvula ou no $A E$, deve-se avaliar funcionalmente as estruturas móveis da prótese e o potencial emboligênico dos trombos (Tabela 20).
Tabela 19 - Recomendações da ecocardiografia na endocardite infecciosa em pacientes com próteses valvares

\begin{tabular}{lc}
\hline Recomendações & Classe \\
\hline $\begin{array}{l}\text { Detecção e caracterização da lesão valvar e avaliação da } \\
\text { gravidade hemodinâmica e performance ventricular* }\end{array}$ & । \\
\hline $\begin{array}{l}\text { Detecção de possíveis complicações, como abscessos, rupturas, } \\
\text { fístulas etc. }\end{array}$ & । \\
\hline Reavaliação nos casos com má evolução clínica* & । \\
\hline Suspeita de endocardite em paciente com culturas negativas* & Ila \\
\hline Bacteremia de etiologia desconhecida* & Ilb \\
\hline Febre persistente sem evidência de bacteremia ou novo sopro*
\end{tabular}

${ }^{*} E T E$ pode dar informações adicionais às obtidas com o ETT.

Tabela 20 - Recomendações da ecocardiografia em pacientes com suspeita clínica de trombose das próteses valvares

\begin{tabular}{lc}
\hline Recomendações & Classe \\
\hline $\begin{array}{l}\text { Avaliação com ETT para determinar as alterações hemodinâmicas } \\
\text { causadas pela trombose }\end{array}$ & Ilb \\
\hline $\begin{array}{l}\text { Realização do ETE para complementar o ETT, avaliar a mobilidade e } \\
\text { potencial emboligênico dos trombos e estudo funcional da prótese }\end{array}$ & Ilb \\
\hline
\end{tabular}

\section{Referências}

1. Cheitlin MD, AlpertJS, Armstrong WF, Aurigemma GP, Beller GA, Bierman FZ, et al. ACC/AHA guidelines for the clinical application of echocardiography: executive summary. J Am Coll Cardiol. 1997; 29: 862-79.

2. ACC/AHA 2006 guideline for the manegement of patients with valvular heart disease. J Am Coll Cardiol. 2006; 48: e1-148.

3. Rahimtoola SH. The problem of valve prosthesis-patient mismatch. Circulation. 1978; 58: 20-4

4. Yoganathan AP, Travis BR. Fluid dynamics of prosthetic valves. In: Otto
CM, editor. The practice of clinical echocardiography, Philadelphia: WB Saunders; 2002. p. 501-24

5. Cosmi JE, Tunick PA, Kronzon I. Mortality in patients with paravalvular abscess diagnosed by transesophageal echocardiography. I Am Soc Echocardiogr. 2004: 17 (7); 766-8.

6. Habib G, Cornen A, Mesana T, Monties JR, Djiane P, Luccioni R. Diagnosis of prosthetic heart valve thrombosis. The respective values of transthoracic and transoesophageal Doppler echocardiography. Eur Heart J. 1993; 14 (4): 447-55 


\section{Diretrizes}

\section{4 - Endocardite infecciosa}

Endocardite infecciosa (EI) é a infecção do endocárdio vascular ou cardíaco resultante da invasão de micro-organismos. Apesar dos avanços nas técnicas diagnósticas e no tratamento, a mortalidade da El permanece elevada ${ }^{1}$. O perfil de apresentação da doença se modificou ${ }^{2}$, com emergência de novos grupos de risco e de micro-organismos mais virulentos, com os estafilococos despontando como principais agentes etiológicos.

O ecocardiograma é fundamental na abordagem da $\mathrm{El}^{3-5}$. A melhor resolução dos aparelhos e, principalmente, o uso da ETE, são responsáveis pela alta acurácia do método no diagnóstico e na avaliação das complicações. O valor adicional da ETE quando o ETT não é diagnóstico está bem definido na alta suspeita clínica de endocardite ou na presença de próteses valvares. Entretanto, a indicação de ETE como exame inicial necessita ser validada por novos estudos ${ }^{5}$.

O diagnóstico definitivo de El baseia-se nas hemoculturas positivas e/ou achados ecocardiográficos característicos. Três achados ao ecocardiograma constituem critérios maiores para o diagnóstico: vegetação definida por uma massa ecodensa móvel, aderida ao endocárdio valvar, mural ou em material protético implantado; abscessos ou fístulas; e nova deiscência de prótese, principalmente quando ocorre tardiamente após o seu implante.

\section{Tabela 21 - Recomendações da ecocardiografia na El}

\begin{tabular}{|c|c|}
\hline Recomendações & Classe \\
\hline \multicolumn{2}{|l|}{ Indicações de ecocardiograma transtorácico (ETT) } \\
\hline Suspeita clínica em pacientes de risco para El com febre sem causa aparente há mais de 48 horas & 1 \\
\hline Quantificar lesões valvares em pacientes com diagnóstico de El & 1 \\
\hline Avaliação inicial das complicações da El & I \\
\hline Reavaliação em pacientes de alto risco (micro-organismos virulentos, piora clínica, febre persistente ou recorrente, novo sopro ou bacteremia persistente) & 1 \\
\hline Avaliação de bacteremia persistente não estafilocócica quando a fonte não é conhecida & Ila \\
\hline Avaliação de febre transitória sem evidências de bacteremia ou novo sopro & III \\
\hline Reavaliação em pacientes com El em valva nativa não complicada & III \\
\hline \multicolumn{2}{|l|}{ Indicações de ecocardiografia transesofágica (ETE) } \\
\hline Quadro clínico sugestivo de endocardite e ETT subótimo & 1 \\
\hline Alta suspeita clínica de El e ETT negativo & I \\
\hline Repetir ETE dentro de 48 horas a uma semana diante da alta suspeita clínica e ETE inicial negativo & I \\
\hline Diagnóstico de El em próteses valvares e na avaliação de complicações & I \\
\hline Endocardite de valva aórtica & 1 \\
\hline Avaliação de complicações da El com potencial impacto no prognóstico e na conduta, como abscessos, perfurações e shunts & I \\
\hline Antes da cirurgia cardíaca, quando realizada na fase aguda de El & I \\
\hline ETE intraoperatória na cirurgia valvar por El & 1 \\
\hline Bacteremia estafilocócica persistente com fonte não identificada & Ila \\
\hline Pode ser considerado na presença de bacteremia estafilocócica nosocomial & $\| l b$ \\
\hline
\end{tabular}

\section{Referências}

1. Nunes MCP, Gelape CL, Ferrari TAC. Profile of infective endocarditic in a tertiary care center in Brazil during a 7-year period: prognostic factors and in hospital outcome. Int J Infect Dis. 2009.

2. Barbosa MM. Endocardite infecciosa: perfil clínico em evolução. Arq Bras Cardiol. 2004; 83: 189-90.

3. Cheitlin MD, Armstrong WF, Aurigemma GP, Beller GA, Bierman FZ, Davis $J \mathrm{~L}$, et al. ACC/AHA/ ASE 2003 guideline update for the clinical application of echocardiography: summary article: a report of the American College of Cardiology/American Heart Association Task Force on Practice Guidelines (ACC/AHA/ASE Committee to Update the 1997 Guidelines for the Clinical Application of Echocardiography). J Am Coll Cardiol. 2003; 42: 954-70.

4. Horstkotte D, Follath F, Gutschik E, Lengyel M, Oto A, Pavie A, et al; Task Force Members on Infective Endocarditis of the European Society of
Cardiology, ESC Committee for Practice Guidelines (CPG); Document Reviewers. Guidelines on prevention, diagnosis and treatment of infective endocarditis executive summary; the task force on infective endocarditis of the European society of cardiology. Eur Heart J. 2004; 25: 267-76.

5. Bonow RO, Carabello BA, Chatterjee K, de Leon AC Jr, Faxon DP, Feed MD, et al. 2008 focused update incorporated into the ACC/AHA 2006 guidelines for the management of patients with valvular heart disease: a report of the American College of Cardiology/American Heart Association Task Force on Practice Guidelines (Writing Committee to revise the 1998 guidelines for the management of patients with valvular heart disease). Endorsed by the Society of Cardiovascular Anesthesiologists, Society for Cardiovascular Angiography and Interventions, and Society of Thoracic Surgeons. J Am Coll Cardiol. 2008; 52: e1-142. 